\title{
Choice and place of death: individual preferences, uncertainty, and the availability of care
}

\author{
Daniel Munday ${ }^{1} \quad$ Jeremy Dale ${ }^{2} \quad$ Scott Murray ${ }^{3}$
}

J R Soc Med 2007; 100:211-215

\section{INTRODUCTION: CHOICE IN A CONSUMER SOCIETY}

Never before have people been faced with such levels of choice as now enjoyed by those living in affluent societies. Increases in technological possibilities and relatively high levels of disposable income have enabled large numbers of citizens to exercise choice in a wide variety of areas, from contraception to managing their infertility, from deciding where to educate children to which career to follow, which TV/DVD/computer to buy, where to holiday, etc.

However, when it comes to deciding about death, choice is a more contentious notion. As the comedian Woody Allen once said 'I am not afraid of death, I just don't want to be there when it happens.' While individuals can influence their health and illness through lifestyle choices, most have relatively little choice with respect to the time, manner and place of their death. ${ }^{1}$ With recent moves to legalize euthanasia and physician-assisted suicide in some countries, choice regarding place and time of death has been extended. However, even where such choice is available, it tends to be utilized by relatively few people. Less than four percent of deaths in the Netherlands are reported to be by euthanasia. ${ }^{2}$

Although most people say that they would prefer to die at home, there has been a persistent fall in the proportion of people doing so. This trend has continued, with only $22 \%$ of cancer deaths in the UK in 2003 occurring there. ${ }^{3}$ The reasons for this are undoubtedly complex, involving many aspects of health-care practice and provision as well as social changes. ${ }^{4}$ The trend in falling home death rate has continued despite the rise in community palliative care services in the UK over the last 20 years.

In line with the all-pervading choice agenda, health care professionals are increasingly expected to ascertain a patient's choice about preferred place of death, record it and aim to deliver it. Little is known about how health-care professionals elicit patients' preferences and the extent to

\footnotetext{
${ }^{1}$ Consultant/Honorary Clinical Senior Lecturer and ${ }^{2}$ Professor of Primary Care, Centre for Primary Health Care Studies, Warwick Medical School, University of Warwick, Coventry CV4 7AL; UK; ${ }^{3}$ St Columba's Hospice Professor of Primary Palliative Care, Division of Community Health Services, University of Edinburgh, Edinburgh EH8 9DX, UK

Correspondence to: Daniel Munday

E-mail: d.munday@warwick.ac.uk
}

which eliciting such views enables them to facilitate their realization. In this paper we present an overview of the UK policy context and the published literature on preferred place of death, and examine key issues which arise for health-care professionals in exploring preferred place of death with patients. The purpose is to inform debate about the role health-care professionals play in helping patients make this most important of choices, and in enabling their preferences regarding preferred place of death to be respected.

\section{CHOICE OF PLACE OF DEATH}

With the recent White Paper Building on the best: choice, responsiveness and equity in the NHS, the UK government's agenda of choice across the spectrum of health-care, from birth to death, was laid out. ${ }^{5}$ With regard to choice and dying it proposed, 'To offer all adult patients nearing the end of life, regardless of their diagnosis, the same access to high quality palliative care so that they can choose if they wish to die at home.' In addition, through funding the End of Life Care Programme, the government specifically sought to implement this policy objective. ${ }^{6}$ Since April 2006, within the Quality and Outcomes Framework, additional payments are payable to GPs for keeping a register of palliative care patients and meeting regularly to plan care at which 'preferred place of care' for individual patients should be noted. ${ }^{7}$

For choice to be meaningful, it must involve at least two-preferably a range of-available, high quality options; which in terms of place of death should arguably include home, hospice, hospital and nursing home. In addition, enabling patients to die in their place of choosing depends on health-care providers understanding the patient's prognosis, likely mode of death, and the availability and accessibility of services. They will then be in a position to assist individual patients and their carers articulate their preferences so that plans can be made and reviewed over time, to enable choices to be turned into realities when the time comes.

\section{PREFERRED PLACE OF DEATH}

Between 50 and $90 \%$ of patients with cancer, when they express a wish concerning place of death, would choose 
to die at home. ${ }^{8}$ Several studies from a variety of countries have linked patients' expression of their preferred place with actual place of death, showing more patients achieve a home death if they have expressed a wish to do so. ${ }^{9-12}$ In addition, patients and carers agreeing on home as the preferred place of death has been shown to be a strong predictive factor in achieving a home death. ${ }^{12}$

Seventeen factors strongly associated with place of death have recently been reported by Gomes and Higginson. ${ }^{13}$ Based on a review of 58 studies, they suggested a conceptual model for ordering such factors in terms of illness, personal and environmental issues, also highlighting the dynamic nature of the model since all factors interact with each other. Preferred place of death is recognized as changing throughout the disease trajectory, with some studies showing that a preference for home death decreases over time, ${ }^{14,15}$ whilst home remained the preferred place given more favourable conditions. ${ }^{15}$

The pattern of change over time was studied qualitatively by Thomas et al. in an interview study of cancer patients and their informal carers. This highlighted the complexities surrounding the issue of preferred place of death. Contextual factors that influenced patients' choice included extent of social network, perception of carers' attitudes, symptom management, fear of loss of dignity and the views of the patient/carers of the care provided by available hospices, nursing homes, hospitals and community nursing and social services. Patients' choices were characterized by uncertainty. Preferences were rarely categorical, but were qualified by speculation about the unpredictability of events. ${ }^{16}$

Although the same standards of care should be offered to all patients with end stage illness, ${ }^{17}$ as is common with issues relating to end of life care the majority of studies into preferred place of death have focused on patients with cancer. ${ }^{8}$ Available evidence suggests that the preferences of patients with other illnesses, and the factors influencing them, are not dissimilar from those with cancer. In a qualitative study of elderly patients' views regarding home as a place of care when dying, concerns were expressed regarding the quality of care which could be provided at home, the appropriateness of their children providing intimate care, and ambivalence towards having professional carers in the home. ${ }^{18}$ One telephone survey of elderly patients who had recently been admitted to hospital with cardiac failure, chronic obstructive airways disease or pneumonia found that approximately equal numbers expressed preferences for home compared with hospital care. ${ }^{19}$ In a follow-up interview study with a selection of these patients, familiar themes regarding not wanting to be a burden on family carers emerged.

\section{DETERMINING PREFERRED PLACE OF DEATH}

It has been suggested that enabling people to die in their preferred place requires greater empowerment of patients and families, early and continuous risk assessment, and better training in palliative care for health-care professionals, including those that work in primary care. ${ }^{9}$ However, there is very little evidence to inform how this should best be done. Issues regarding preferred place of death will often be discussed along with other concerns of the patient, including questions regarding likely prognosis and mode of death. The difficulty in predicting prognosis and mode of death may confound such discussion. The very concept of a terminal phase with non-cancer patients is problematic with, for example, patients with end stage heart failure or chronic obstructive airways disease tending to follow a trajectory of steady decline with episodes of acute deterioration and recovery, rather than the relatively rapid decline as tends to occur for cancer patients with advanced metastatic disease (Box 1). ${ }^{20}$ Whilst determining prognosis for cancer patients is problematic, with a tendency for overestimation of survival, ${ }^{21}$ clinical prediction criteria for prognosis in patients with end stage non-cancer diagnosis have been shown to be ineffective; ${ }^{22}$ in addition, one study showed that primary care physicians tended to over-estimate the likelihood of death at one year in patients with end stage heart failure. ${ }^{23}$

Each individual patient's expectations of the dying process and the way that this shapes their wishes is unique. Helping patients to identify and articulate their preferences, and how these evolve in response to the patient's changing condition, requires well-developed communication skills, including considerable sensitivity to differences in values. Such discussions can only take place effectively within the context of an ongoing and trusting patient-clinician relationship, and may require a considerable investment of time. ${ }^{25}$

The extent to which patients wish to discuss their preferred place of death varies from individual to individual. While some will have openly acknowledged that they are dying, may be clear of their wish and will articulate clearly their preferred place of death without being prompted, others will be in denial regarding their terminal illness, using this as a protective mechanism; the subject of where they wish to die may be off limits. Still other patientspossibly the majority — will be ambivalent. They may not voice their concerns or wishes openly but may be ready to discuss the issue if sensitively broached. They may be clear of what they would want in optimal circumstances, but may recognize that circumstances as they approach the time of dying may not favour this and that an alternative place of death might occur. 
Three end of life illness trajectories have been described, although in practice there is often much overlap between them for individual patients, and death can occur at any point along the course of the illness. ${ }^{20}$

(1) Long period of chronic illness and short period of terminal decline

- Typically occurs in cancer;

- The onset of terminal decline may be relatively clear, although length of the process is difficult to predict;

- Even within the terminal phase, there may be periods of improvement and deterioration with high degrees of individual variation.

(2) Gradual functional deterioration punctuated by episodes of serious acute illness

- Typically occurs with advanced cardio-respiratory disease;

- Functional impairment secondary to reduction in cardiorespiratory performance is relentless, often over several years;

- Acute exacerbations of heart failure or acute on chronic respiratory failure punctuate this course, each one of which is potentially fatal, although typically several occur before the final attack ensues;

- Functional impairment following exacerbations is frequently marked;

- Patients in this group often have co-morbid conditions.

\section{(3) 'Prolonged dwindling' of the very elderly}

- Often seen in residents of care homes for the elderly;

- Patients frequently have multiple degenerative disorders, including cognitive impairment resulting from various forms of dementia;

- The patient suffers poor health and low levels of functioning over a prolonged period of time, sometimes several years;

- The terminal event may be the result of an acute problem, such as chest infection or fractured neck of femur, or may be harder to define in terms of medical causation.

The patient's and carer's wishes may vary with time and within different contexts, including the extent to which active treatment is still felt to be an option. For example, a patient may become fearful of being at home because of a lack of support, or symptoms may arise which might be more easily managed as an inpatient. Preferred place of death should therefore be seen as a dynamic issue within which the patient's wishes fluctuate over time. With all of the contingent issues, the outcome of discussions of preferred place of death may not be a definitive answer of 'home' or 'hospice', but might, as Thomas et al. suggest, reveal 'a stronger or weaker leaning in one direction.' 16

\section{ADVANCE CARE PLANNING ${ }^{26}$}

Knowledge of preferences will aid advance care planning for both cancer ${ }^{27}$ and non cancer patients ${ }^{17,28}$ and should help to avoid inappropriate management, particularly hospital admission, at the end of life (Box 2). Informing secondary care providers of a patient's wish to die at home can enable forward planning so that discharge can be arranged at short notice if necessary. However, the imminence of death is often difficult to recognize, and problems with estimating prognosis may have the consequence that hospital admission (e.g. for symptom control), although intended to be shortterm, will result in the patient not being discharged. This may be especially problematic in non-cancer diagnoses, where prognosis is even more difficult to predict. While further research may enable new models which improve accuracy of prediction for both cancer and non-cancer diseases to emerge, ${ }^{29,30}$ given the non-linear complexity of terminal illness, the precision of such models will always have its limitations. ${ }^{31}$

\section{EFFECTS ON THE HEALTH-CARE PROFESSIONAL}

Little is known about the effect on health-care professionals of discussing preferred place of death. The process may stimulate negative emotions, with concerns that the patient will react emotionally to the subject being broached. They may have personal fears regarding discussing such a difficult and emotive subject openly, including uncertainty about how best to handle the patient's and carer's feelings, or worries about being asked to make assurances which they may not be able to fulfil. They may have concerns about mortality for themselves or their family and friends, or they may have unresolved grief from past bereavements which makes the subject of death particularly difficult to discuss. Personal knowledge of the patient over time may help to prepare the professional and the patient for discussion of the issue, but conversely it may also make the issue more difficult to discuss openly if an emotional tie has developed between them. Professionals with a close relationship with patients may tend to overestimate prognosis and fail to recognize the impending onset of the terminal phase. ${ }^{32}$

The health-care professional may feel it particularly difficult to discuss preferred place of death with a patient when providing adequate community services or securing admission to a hospice may not be possible. Preferred place of death may be a hollow concept and the promise of choice a cruel sham if services are not available because of lack of funding or other resources. Whilst simplistic rhetoric promising patients a planned death is seductive, the reality may be that the complex uncontrollable nature of the dying process exposes real choice as being fool's gold.

\section{CONCLUSION}

In this paper, we have summarized issues that influence choice and preferred place of death. Ascertaining a patient's 


\section{Case 1: Mr A, a 76-year-old ex-lorry driver with NYHA stage IV heart failure, diabetes, painful neuropathy of his lower legs and gout in his feet.}

Mr A is housebound and cared for by his 69-year-old wife, who is in reasonable health. He has had several admissions to hospital with acute left ventricular failure (LVF) over the last two years, which he always finds distressing. He discusses future management with his cardiac failure nurse on one of her regular visits, when he says he has 'had enough.' They explore alternatives to hospital admission for any further episode of acute LVF, and Mr A agrees to a multidisciplinary discussion of plans for managing him at home if at all possible. This discussion takes place at the next primary care team meeting between the GP and district nurse which the cardiac failure and Macmillan nurse attend.

A plan is drawn up for Mr A to have subcutaneous diamorphine via a syringe driver and furosemide as required at home if and when further LVF occurs. The cardiac failure nurse checks the appropriate dose of furosemide with his cardiologist and the Macmillan nurse and GP decide on the appropriate dose of diamorphine. A prescription for the agreed drugs is written so that anticipatory medication can be kept in his home. Instructions are written in the district nursing notes kept in his home and faxed to the out-of-hours primary care provider.

Six weeks later Mr A suffers breathlessness and acute chest pain on a Sunday afternoon. He is visited by the on-call GP who gives him intravenous furosemide and arranges for the district nurse to commence the diamorphine driver.

All has gone to plan but the outcome is still not certain. The following two scenarios are amongst several possibilities:

Scenario A: Mr A's pain is controlled and his breathlessness improves. Mr A becomes unconscious in the evening and dies the early hours of the next morning, surrounded by his family.

Scenario B: With controlled pain and improved breathlessness, Mr A remains bed-bound for several days, but with review by the cardiac failure nurse who receives telephone advice from the cardiologist. His ACE inhibitor dose is adjusted and he recovers to the level of his previous functioning, though he remains frustrated at his poor quality of life.

\section{Case 2: Mr B, a 63 year old retired headmaster with lung cancer}

Mr B has discussed his wish to die at home with his GP since early after his diagnosis with advanced disease six months ago. He has had two admissions with shortness of breath, for which he has had a right pleural tap and a transfusion on one occasion. He has been increasingly short of breath for a week and the district nurse takes blood for a full blood count. His haemoglobin is 7.6. His GP visits and discovers that he also has reduced air entry in his right lung base. They discuss the situation and Mr B agrees that he should be admitted onto the oncology ward for a transfusion and possible pleural tap. On admission an ultrasound scan of his chest reveals mostly solid tumour with a small amount of loculated fluid in his right chest. Transfusion is arranged for three units of blood.

Despite transfusion the breathlessness increases and $\mathrm{Mr} \mathrm{B}$ becomes quite distressed. He is using continuous oxygen, and a syringe driver with diamorphine and midazolam sedation is commenced. He settles to some extent but is quite drowsy and confused. His wife and family feel that they could not cope even with additional support at home and request that he remains in hospital. Mr B is too confused and drowsy to enter into discussions. He dies in hospital after a further 48 hours.

The Macmillan nurse spends time with Mrs B exploring her feelings with regard to her husband's wishes for a home death not being met. She is upset and feels that she has failed him. Several meetings are needed to support Mrs B.

Finally she accepts the inevitability of the hospital death, but still feels sad that her husband did not die at home as he had hoped.

wishes with regard to place of death is an important function in end of life care, and should be approached sensitively and regularly reviewed as the patient approaches death, within a realistic evaluation of the feasibility of different options. Discussions will need to involve the patient's family and/or other informal carers. Preferences when clarified can aid in advance care planning to enable patients to achieve their goals; however, the complexities surrounding clinical course and limited resources are likely to confound patients' plans even with health-care services of the highest standard.

Since the issue is difficult to discuss, a professional with or district nurse - is arguably the most appropriate person to perform the task, using a sensitive approach, well developed communication skills and providing ongoing relational continuity. Whilst clearly recording the patient's preference in the health-care record is important, it must not become a mechanical 'tick box' exercise. The recent change to the Quality and Outcomes Framework in the UK has introduced a financial incentive for practitioners to record patients' preferred place of death. While this may encourage more patient-centred end of life-care, perversely it could lead to a more perfunctory approach. Investigating the experiences of health-care professionals in this difficult and sensitive task is an important area for further research. 


\section{REFERENCES}

1 Drought TS, Koenig BA. 'Choice' in end-of-life decision making: researching fact or fiction? Gerontologist 2002;42:114-28

2 Onwuteaka-Philipsen B, van der Heide A, Koper D, et al. Euthanasia and other end-of-life decisions in the Netherlands in 1990, 1995, and 2001. Lancet 2003;362:395-9

3 ONS. Mortality Statistics: General. DH1 No 362003 [Web Page]. 2005; Available at http://www.statistics.gov.uk/downloads/theme_health/ Dh1_36_2003/DH1_2003.pdf (accessed 08/03/2006)

4 Clark D, Seymour J. Reflections on Palliative Care: Sociological and Policy Perspectives. Buckingham: Open University Press, 1999

5 DOH. Building on the Best: Choice, Responsiveness and Equity in the NHS. London: Department of Health, 2003

6 End of Life Care Programme [Web Page]. Available at http:// eolc.cbcl.co.uk/eolc (accessed 15/03/2006)

7 BMA. Focus on the Quality and Outcomes Framework [Web Page] 2006. Available at http://www.bma.org.uk/ap.nsf/Content/ focusqoffeb06 (accessed 10/05/2006)

8 Higginson I, Sen-Gupta. Place of care in advanced cancer: A qualitative systematic literature review of patient preferences. J Palliat Med 2000; 3:287-300

9 Karlsen S, Addington-Hall J. How do cancer patients who die at home differ from those who die elsewhere? Palliat Med 1998;12:279-86

10 Fukui S, Kawagoe H, Masako S. Determinants of the place of death among terminally ill cancer patients under home hospice care in Japan. Palliat Med 2003;17:445-53

11 Brazil K, Bedard M, Willison K. Factors associated with home death for individuals who receive home support services: a retrospective cohort study. BMC Palliat Care 2002;1:1-6

12 Cantwell P, Turco S, Brenneis C, Neuman C, Bruera E. Predictors of home death in palliative care cancer patients. J Palliat Care 2000;16: 23-8

13 Gomes B, Higginson I. Factors influencing death at home in terminally ill patients with cancer: systematic review. BMJ 2006;332:515-21

14 Hinton J. Can home care maintain an acceptable quality of life for patients with terminal cancer and their relatives? Palliat Med 1994;8: 183-96

15 Townsend J, Frank A, Fermont D, et al. Terminal cancer care and patients' preference for place of death: a prospective study. BMJ 1990; $301: 415-7$
16 Thomas C, Morris S, Clark D. Place of death: preferences among cancer patients and their carers. Soc Sci Med 2004;58:2431-44

17 Traue DC, Ross JR. Palliative care in non-malignant diseases. J Roy Soc Med 2005;98:503-6

18 Gott M, Seymour J, Bellamy G, Clark D, Ahmedzai S. Older people's views about home as a place of care at the end of life. Palliat Med 2004; 18:460-7

19 Fried TR, Doorn CV, O'Leary JR, Tinetti ME, Drickamer MA. Older persons' preferences for site of terminal care. Ann Intern Med 1999; 131:109-12

20 Murray S, Kendall M, Boyd K, Sheikh A. Illness trajectories and palliative care. BMJ 2005;330:1007-11

21 Glare P, Virik K, Jones M, et al. A systematic review of physicians' survival predictions in terminally ill cancer patients. BMJ 2003;327: 195-201

22 Fox E, Landrum-McNiff K, Zhong Z, Dawson NV, Wu AW, Lynn J. Evaluation of prognostic criteria for determining hospice eligibility in patients with advanced lung, heart, or liver disease. JAMA 1999;282: $1638-45$

23 Muntwyler J, Abetel G, Gruner C, Follath F. One-year mortality among unselected outpatients with heart failure. Eur Heart J 2002;23: 1861-6

24 Lunney JR, Lynn J, Foley DJ, Lipson S, Guralnik JM. Patterns of functional decline at the end of life. JAMA 2003;289:2387-92

25 Thomas $C$. The place of death of cancer patients: can qualitative data add to known factors? Soc Sci Med 2005;60:2597-607

26 Murray S, Sheikh A, Thomas K. Advance care planning in primary care. BMJ 2006;333:868-9

27 NICE. Guidance on Cancer Services: Improving Supportive and Palliative Care for Adults. London: National Institute for Clinical Excellence, 2004

28 Davison S, Simpson C. Hope and advance care planning in patients with end stage renal disease: qualitative interview study. BMJ 2006; 333:886-9

29 Cowie MR. The fine art of prognostication. Eur Heart J 2002;23: $1804-6$

30 Parkes CM. Prognoses should be based on proved indices not intuition. BMJ 2000;320:473

31 Munday D, Johnson S, Griffiths F. Complexity theory and palliative care. Palliat Med 2003;17:308-9

32 Christakis N, Lamont E. Extent and determinants of error in doctors prognosis in terminally ill patients: prospective cohort study. BMJ 2000;320:469-73 